\title{
Eficiência das lnstituições de Microcrédito: uma aplicação de DEA/VRS no contexto brasileiro
}

\author{
Elaine Aparecida Araújo ${ }^{\mathrm{a} *}$, Charles Ulises de Montreuil Carmona ${ }^{\mathrm{b}}$ \\ a*Universidade Federal Fluminense, Niterói, RJ, Brasil, elaineadc13@gmail.com \\ bUniversidade Federal de Pernambuco, Recife, PE, Brasil.
}

\begin{abstract}
Resumo
0 objetivo do presente artigo foi avaliar a eficiência de instituições de microcrédito brasileiras sob dois enfoques distintos: financeiro e social. Em termos metodológicos, foi utilizada a técnica de programação linear Data Envelopment Analysis - DEA com retornos variáveis de escala (DEA/VRS) aplicada sobre uma amostra de instituições listadas na base THE MIX em 2008, 2009 e 2010; e o Índice de Malmquist para análise das variações de produtividade e eficiência ao longo do tempo. Os resultados evidenciam maiores escores de eficiência social comparativamente à financeira para cada ano individualmente. A análise temporal revelou aumento da produtividade sob ambas as abordagens. Esse aumento refletiu variações efetivas da eficiência técnica, em maior grau, no modelo social, e deslocamentos da fronteira tecnológica no modelo financeiro. De modo geral, esses resultados sinalizam uma maior homogeneidade das instituições no que se refere a medidas e práticas relacionadas à eficiência social comparativamente à financeira.
\end{abstract}

Palavras-chave

Finanças. Microcrédito. Desenvolvimento econômico. Eficiência. Data Envelopment Analysis.

\section{Introdução}

Esse artigo se volta para a avaliação de desempenho de instituições de microcrédito brasileiras. 0 desempenho é aqui tratado sob uma ótica mais restrita, tomando-se a eficiência institucional como o principal aspecto de análise.

Em termos explícitos, o objetivo principal do presente artigo é mensurar e avaliar a eficiência das instituições de microcrédito brasileiras sob o enfoque financeiro e social, empregando, para tal, a técnica Data Envelopment Analysis - DEA. A eficiência, aqui entendida em seu sentido econômico, denota quão bem a entidade de microcrédito analisada está empregando recursos para gerar outputs ou resultados vinculados aos seus objetivos organizacionais. A escolha da $D E A$ como técnica de análise se justifica pelo fato dela permitir que diversas variáveis ou dimensões sejam agregadas em um indicador único que evidencia o nível de eficiência institucional sob a perspectiva analisada.

De modo a abarcar os princípios das duas perspectivas filosóficas existentes na literatura sobre a natureza das instituições financeiras (abordagem de intermediação e de produção; Berger \& Humphrey, 1997) e, principalmente, considerando as peculiaridades das instituições microfinanceiras, optou-se, neste trabalho, pela aplicação da $D E A$ com duas óticas de análise distintas: financeira e social. A abordagem financeira é delineada de modo a aferir a eficiência das instituições na geração de receitas financeiras e ampliação do volume de recursos em suas carteiras ativas. Já a social leva em conta, principalmente, o número de clientes atendidos e a profundidade do alcance social da instituição, variável que representa a situação socioeconômica/nível de pobreza do público atendido.

Acredita-se que diante desse duplo caráter peculiar às instituições microfinanceiras, a avaliação de desempenho dessas instituições deve ser norteada, tanto por elementos financeiros quanto por aqueles de caráter social. Os resultados alcançados por essas instituições em ambos os campos (financeiro e social) são igualmente importantes para seu êxito e para o efetivo alcance de sua missão social. 
No contexto específico das instituições de microfinanças, as abordagens dedicadas à avaliação do desempenho surgidas recentemente estão ancoradas na realidade internacional, o que denota a necessidade de exploração dessa questão no Brasil, principalmente tendo em conta a importância do microcrédito no âmbito socioeconômico e de políticas públicas. Acrescente-se a isso o fato de as metodologias já construídas emergirem de um contexto específico relativo a uma instituição ou agência de rating, tornando delicada a sua aplicação generalizada a outras realidades. Tomados conjuntamente, esses fatores justificam a importância de uma abordagem de avaliação de desempenho de instituições de microcrédito dessa natureza, em âmbito nacional, propósito a que se debruça o presente artigo.

\section{Referencial teórico}

\subsection{Microcrédito e microfinanças}

0 microcrédito e as microfinanças despontaram no rol de alternativas para a solução do problema de exclusão ou restrição de acesso a serviços creditícios e financeiros por parte de alguns segmentos como população de baixa renda, microempreendimentos formais e informais, pequenas empresas etc. (Braga $\&$ Toneto Junior, 2000).

Barone et al. (2002) e Naqvi \& Guzmán (2003/2004) definem microfinanças como um conjunto de serviços financeiros (poupança, créditos, seguros etc.), prestados por instituições financeiras ou não, para indivíduos de baixa renda e microempreendimentos (formais e informais) excluídos (ou com acesso restrito) ao sistema financeiro tradicional.

Segundo Soares \& Melo Sobrinho (2008), do total da população mundial considerada carente, mais de 500 milhões são economicamente ativos. São pessoas que ganham a vida trabalhando por conta própria em microempreendimentos (negócios muito pequenos) e, geralmente, não têm acesso a serviços financeiros adequados e sustentáveis. Foi para preencher essa lacuna e atender a esse "nicho" de mercado que surgiu a indústria microfinanceira, focada na concessão de serviços financeiros especializados a pessoas de baixa renda.

No Brasil, a parte mais visível e desenvolvida do complexo conjunto de ferramentas microfinanceiras de geração de renda e combate à pobreza é o microcrédito (Parente, 2002), que é conceituado, segundo Nichter et al. (2002, p. 15) como "[...] a concessão de empréstimos de pequeno valor, para atividade produtiva, no contexto das microfinanças". Alves \& Soares (2004, p. 9) apresentam definição semelhante. Para eles,
[...] a atividade de microcrédito é definida como aquela que, no contexto das microfinanças, dedica-se a conceder crédito para o financiamento de pequenos empreendimentos e diferencia-se dos demais tipos de empréstimo essencialmente pela metodologia utilizada, bastante diferente daquela adotada para as operações de crédito tradicionais, à medida que envolve o emprego de agentes de crédito, garantias solidárias e empréstimos com prazos curtos e valores crescentes.

0 termo microcrédito é empregado com vários significados na literatura, indicando, muitas vezes, produtos e formas de concessão de crédito distintos. Desta forma, é importante apresentar os principais aspectos que caracterizam o microcrédito como uma modalidade específica de crédito, qual seja, o crédito produtivo e orientado, conforme explicitado em Alves \& Soares (2004), Barone et al. (2002) e Ribeiro \& Carvalho (2006):

- Crédito produtivo: o microcrédito visa apoiar atividades produtivas de negócios de pequeno porte, mantidos por pessoas de baixa renda, não se destinando, portanto, ao financiamento do consumo;

- Crédito orientado: acompanhamento dos créditos é realizado pelo agente de crédito (funcionário da instituição), que é o profissional que desempenha o papel de acompanhar o tomador antes, durante e depois de contraído o empréstimo;

- Sistema de garantias: uso do aval ou fiança solidária, que consiste na formação de grupos de tomadores que se responsabilizam solidariamente pelo compromisso com a instituição, e assumem coletivamente as responsabilidades pelos créditos concedidos a cada um dos componentes do grupo.

\subsection{Objetivos financeiro e social e avaliação de desempenho no microcrédito}

0 entusiasmo com o microcrédito e a atenção que ele desperta atualmente tem origem no fato de seus princípios originais e sua idéia fundamental residirem no combate à pobreza através da geração de trabalho e renda, com conseqüente melhoria das condições sociais e econômicas dos clientes (Yunus, 2002), o que confere a esse tipo de crédito um caráter reconhecidamente social.

Embora se diferenciem de instituições financeiras tradicionais, as instituições de microcrédito possuem também objetivos, características e estratégias de natureza financeira, como qualquer outra entidade que realiza atividade de intermediação financeira. Destarte, elas necessitam adotar estratégias de gestão focadas em resultados financeiros positivos e eficiência financeira, sem perder de vista o foco social. 
Várias são as discussões na literatura sobre a performance institucional no que tange aos objetivos financeiro e social. Duas correntes se destacam: welfaristas e institucionalistas.

Sob a vertente welfarista, os parâmetros principais para avaliação do desempenho da instituição são: alcance social, aferido pelo nível socioeconômico/grau de pobreza da clientela atendida (aspecto denominado profundidade do alcance); impacto socioeconômico, que envolve mensurar como e quanto a instituição contribui para melhorar as condições de vida e aliviar a pobreza. Eles tendem a atribuir mais destaque à profundidade do alcance do que à sua amplitude (número total de clientes atendidos), e avaliam o sucesso da instituição de acordo com tais parâmetros sociais (Ejigu, 2009).

Por outro lado, para os institucionalistas, as organizações devem ser avaliadas pelo seu nível de sustentabilidade e desempenho financeiro, pois apenas instituições financeiramente sustentáveis e eficientes terão condições de concretizar os objetivos sociais de contribuir para redução da pobreza e melhoria das condições socioeconômicas dos clientes. Nesse sentido, os institucionalistas lançam mão de indicadores financeiros para avaliar o desempenho de uma instituição de microcrédito/microfinanças (Ejigu, 2009).

0 presente artigo vislumbra o desempenho institucional sob a ótica financeira e também sob a ótica social. Coaduna, assim, com visões teóricas mais recentes presentes na literatura que reconhecem o desempenho de uma instituição como um fenômeno multifacetado e complexo, que requer abordagens de análise multidimensionais e abrangentes (Carneiro et al., 2005; Kaplan \& Norton, 1997).

\subsection{Conceitos fundamentais relacionados à técnica $D E A$}

Tomando como respaldo o arcabouço teórico construído, principalmente os conceitos econômicos de eficiência propostos por Farrel (1957), a metodologia Data Envelopment Analysis - DEA foi desenvolvida por Charnes et al. (1978), caracterizando-se como uma abordagem não paramétrica para análise de eficiência de unidades produtivas (Mello et al., 2005). Segundo Lins \& Meza (2000), a DEA compara a eficiência de várias unidades similares mediante consideração explícita do uso de suas múltiplas entradas para produção de múltiplas saídas.

Mello et al. (2005) se referem à eficiência técnica como um conceito fundamental em $D E A$, afirmando que ela consiste em comparar o que foi produzido, dado os recursos disponíveis, com o que poderia ter sido produzido com os mesmos recursos. Os conceitos de DMUs, inputs, outputs são também balizadores da DEA. Decision Making Units - DMUs são as unidades cuja eficiência está sendo avaliada. Outputs são os resultados (produtos) obtidos por cada uma das $D M U$ s e os inputs representam os recursos (insumos) consumidos por cada uma das $D M U s$ na obtenção dos resultados desejados.

Dados esses conceitos, pode-se definir a $D E A$, de forma mais detalhada, como um método quantitativo e empírico que mede o desempenho relativo de unidades independentes e semelhantes, gerando um único indicador de desempenho para cada unidade avaliada, a partir da relação ponderada entre insumos e produtos. Em termos técnicos, a $D E A$ se utiliza do processo de otimização da programação linear para construir uma fronteira de produção empírica ou superfície envoltória de máximo desempenho. Isso permite que se identifiquem unidades-referência, cujos índices de desempenho servem como referencial para as demais unidades posicionadas sob a fronteira e caracterizadas como ineficientes (Niederauer, 1998).

De acordo com Lins \& Meza (2000) um grande benefício da $D E A$ é justamente a habilidade em apontar esse conjunto de unidades de referência, que pode ser utilizado como benchmark na melhoria do desempenho das unidades menos eficientes.

A $D E A$ é considerada uma técnica não paramétrica para mensurar a eficiência. Os métodos não paramétricos não requerem a especificação de uma forma funcional para a relação entre as variáveis de insumo e produto, nem fazem suposições sobre o comportamento econômico das empresas cuja eficiência está em análise. A $D E A$, especificamente, considera que o máximo que poderia ser produzido é obtido por meio da observação das unidades mais produtivas (Mello et al., 2005), delineando uma fronteira de melhores práticas a partir das próprias unidades avaliadas (Ferreira \& Gomes, 2009).

São várias as formulações dos modelos de $D E A$ encontradas na literatura. Entretanto, dois modelos básicos de $D E A$ são geralmente usados nas aplicações. 0 primeiro é o modelo original proposto por Charnes et al. (1978), denominado CRS (Constant Returns to Scale), que assume retornos constantes de escala. 0 segundo é o modelo denominado VRS (Variable Returns to Scale), desenvolvido por Banker et al. (1984), utiliza uma formulação que permite retornos crescentes e decrescentes, levando em consideração o tamanho das $D M U$ s avaliadas (Ferreira \& Gomes, 2009; Mello et al., 2005).

No que se refere à orientação, os modelos orientados a inputs estão voltados para a redução de insumos, mantendo-se fixos os níveis de outputs 
(produto). Já os modelos orientados a outputs mantêm fixos os níveis de insumos, centrando-se na expansão do produto.

Os modelos CCR orientados a input e outputs são esboçados a seguir.

Modelo CCR (ou CRS) orientado a inputs

$$
\operatorname{Max} E_{o}=\sum_{j=1}^{s} u_{j} y_{j o}
$$

S.a

$\sum_{i=1}^{r} v_{i} x_{i o}=1$

$\sum_{j=1}^{s} u_{j} y_{j k}-\sum_{i=1}^{r} \mathrm{v}_{\mathrm{i}} x_{i k} \leq 0, \forall k$

$u_{j}, v_{j} \geq 0 \quad \forall i, j$

Modelo CCR (ou CRS) orientado a outputs

$$
\operatorname{Min} \mathrm{h}_{\mathrm{o}}=\sum_{i=1}^{r} v_{i} x_{i o}
$$

S.a

$$
\sum_{j=1}^{s} u_{j} y_{j o}=1
$$

$\sum_{i=1}^{r} \mathrm{v}_{\mathrm{i}} x_{i k}-\sum_{j=1}^{s} u_{j} y_{j k} \geq 0, \forall k$

$$
u_{j}, v_{j} \geq 0 \quad \forall i, j
$$

Nos modelos acima, E é a eficiência da $D M U$ que está sendo avaliada; $v_{i}$ e $u_{i}$ são os pesos dos inputs $i$ $(i=1, \ldots$, r) e outputs $j(j=1, \ldots, s)$ respectivamente; e $\mathrm{y}_{\mathrm{jk}}$ são os inputs $i$ e outputs $j$ da DMU K $(\mathrm{K}=1, \ldots n)$; $\mathrm{x}_{\mathrm{io}}$ e $\mathrm{y}_{\mathrm{jo}}$ são os inputs $i$ e outputs $j$ da $D M U$ em análise. As variáveis de decisão são os pesos $v_{i}$ e $u_{j}$, ou seja, o problema consiste em encontrar pesos que otimizem o índice de eficiência. A estrutura matemática do modelo permite que uma $D M U$ seja considerada eficiente com vários conjuntos de pesos. Em particular, podem ser atribuídos pesos zeros a algum input ou output, o que significa que essa variável foi desconsiderada na avaliação. No modelo CCR orientado a outputs, a eficiência é dada por $1 / h_{0}$ (Cook \& Zhu, 2008; Mello et al., 2005).

Diferentemente dos modelos CCR (ou CRS), os modelos BCC (ou VRS) consideram retornos variáveis de escala, isto é, substituem o axioma da proporcionalidade entre inputs e outputs pelo axioma da convexidade. Assim, ao obrigar que a fronteira de produção seja convexa, o modelo BCC (ou $V R S$ ) permite que as DMUs que operam com baixos valores de inputs tenham retornos crescentes de escala e as que operam com altos valores tenham retornos decrescentes de escala (Mello et al., 2005). Em termos matemáticos, tais especificidades são representadas por uma variável adicional na função objetivo e na segunda restrição $\left(u^{*}\right.$ ou $\left.v^{*}\right)$, variável essa que representa o fator de escala, podendo indicar retornos de escala crescentes, decrescentes ou nulos (Cook \& Zhu, 2008). Os modelos BCC orientados a input e outputs são esboçados a seguir.

Modelo BCC (ou VRS) orientado a inputs

$$
\operatorname{Max} E_{o}=\sum_{j=1}^{s} u_{j} y_{j o}+u^{*}
$$

S.a

$\sum_{i=1}^{r} v_{i} x_{i o}=1$

$\sum_{j=1}^{s} u_{j} y_{j k}-\sum_{i=1}^{r} \mathrm{v}_{\mathrm{i}} x_{i k}+u^{*} \leq 0, \forall k$

$u_{j}, v_{j} \geq 0 \forall i, j, \quad u^{*} \in \Re$

Modelo BCC (ou VRS) orientado a outputs

$\operatorname{Min} \mathrm{h}_{\mathrm{o}}=\sum_{i=1}^{r} v_{i} x_{i o}+v^{*}$

S.a

$\sum_{j=1}^{s} u_{j} y_{j o}=1$

$\sum_{i=1}^{r} \mathrm{v}_{\mathrm{i}} x_{i k}-\sum_{j=1}^{s} u_{j} y_{j k}+v^{*} \geq 0, \quad \forall k$

$u_{j}, v_{j} \geq 0 \forall i, j, \quad v^{*} \in \Re$

\subsection{Análise de eficiência em instituições financeiras e microfinanceiras}

A ampla aplicação da $D E A$ em estudos de avaliação de eficiência de instituições financeiras/bancos convencionais acenou para a possibilidade de emprego dessa técnica também no âmbito da avaliação de entidades de microfinanças, tendo em vista que essas instituições se constituem modalidades específicas pertencentes ao conjunto maior de instituições prestadoras de serviços financeiros.

Annim (2010) define a eficiência no contexto microfinanceiro como a habilidade institucional de utilizar uma combinação ótima de recursos 
(colaboradores, tempo de trabalho, custos operacionais) para gerar o máximo de produto, que está associado, geralmente, ao número de empréstimos e clientes atendidos pela instituição, especialmente aqueles de menor renda.

A esse respeito, Gutiérrez-Nieto et al. (2009) consideram que no microcrédito uma instituição deve ser considerada eficiente se ela obtiver êxito na promoção de objetivos financeiros e sociais. $\mathrm{Ou}$ seja, ela deve ser capaz de escolher uma combinação adequada de inputs/outputs relacionados a ambos objetivos sociais e financeiros, e utilizá-los de maneira eficiente.

Quando se avalia a eficiência de instituições financeiras, existem diferentes perspectivas filosóficas de análise que podem ser utilizadas para mensurar o fluxo de atividades e serviços providos pela instituição (Gutiérrez-Nieto et al., 2007). Duas abordagens são predominantes na literatura: intermediação e produção. Segundo Berger \& Humphrey (1997), o papel funcional de uma instituição financeira pode ser avaliado sob o prisma dessas duas perspectivas.

A abordagem de intermediação considera as instituições financeiras como intermediários entre depositantes e tomadores de empréstimos. 0 papel principal das instituições, sob essa ótica de análise, é transferir recursos financeiros dos agentes superavitários para os agentes deficitários, aprimorando a alocação de fundos na economia (GonzalezVega,1986). Em estudos de eficiência realizados sob o prisma da abordagem de intermediação, depósitos e outros recursos captados pelas instituições são considerados inputs enquanto os valores da carteira de empréstimos são, tradicionalmente, tidos como outputs (Gutiérrez-Nieto et al., 2007).

Por seu turno, a abordagem de produção concebe as instituições financeiras como empresas (fábricas) que se dedicam à produção e prestação de serviços aos seus clientes. Assim, essas instituições utilizam recursos físicos (ativos, capital, trabalho), que são empregados para a geração de serviços e transações financeiras ofertados aos clientes. Os estudos realizados na perspectiva da abordagem de produção geralmente consideram mão de obra e ativos como inputs e o número e tipo de transações efetuadas (basicamente, depósitos e empréstimos) como outputs (GutiérrezNieto et al., 2007).

\section{Metodologia}

\subsection{Coleta de dados e amostragem}

Os dados coletados para esse estudo foram relativos ao desempenho financeiro de um grupo de instituições de microcrédito brasileiras, visando aplicar a técnica de análise $D E A$.
A fonte principal de informações foi a base de dados on line do Microfinance Information Exchange - THE MIX (2010). Essa instituição coleta, organiza e divulga uma ampla gama de informações sobre as instituições provedoras de serviços de microfinanças/microcrédito, tendo como principal instrumento uma plataforma on line. Essa plataforma, desenvolvida e alimentada pela instituição, possui informações sobre desempenho financeiro, relatórios contábil-financeiros e relatórios de auditoria de instituições de microcrédito/microfinanças do mundo inteiro, apresentadas de forma segmentada (escala, região geográfica, etc.).

Os dados coletados foram sistematizados em bases de dados do SPSS - Statistical Package for Social Sciences ${ }^{\circledR}$ e do Frontier Analyst ${ }^{\circledR}$, softwares utilizados, respectivamente, para o desenvolvimento das análises estatísticas preliminares e análise $D E A$.

A coleta de dados abrangeu informações de todas as instituições de microfinanças brasileiras que se encontraram disponíveis na plataforma $T H E$ MIX, referentes aos anos de 2008, 2009 e 2010. A Tabela 1 demonstra o número total de instituições, bem como sua distribuição por formato institucional.

\subsection{Especificação do modelo e definição das variáveis}

Este trabalho optou pelo desenvolvimento de dois modelos ou arranjos diferentes para avaliação da eficiência das instituições de microcrédito. 0 primeiro modelo, aqui denominado modelo de eficiência financeira, é norteado pela abordagem de intermediação e considera que a instituição prima pela maximização de seus resultados financeiros. Assim, ela atuará no sentido de alargar o volume de recursos emprestados e suas receitas financeiras, empenhando-se para obtenção de resultados financeiros positivos, questão primordial para a instituição, segundo o eixo teórico da abordagem de intermediação.

0 segundo modelo, batizado neste trabalho por modelo de eficiência social, está assentado nos pressupostos da abordagem de produção, mas é

Tabela 1. Número de organizações por formato institucional.

\begin{tabular}{lccc} 
& \multicolumn{3}{c}{ Ano } \\
\cline { 2 - 4 } & 2008 & 2009 & 2010 \\
\hline ONGs & 22 & 17 & 14 \\
SCMs & 04 & 03 & 01 \\
Cooperativas/Cooperativas & 03 & 03 & 03 \\
Total & 29 & 23 & 18 \\
\hline
\end{tabular}

Fonte: Dados da Pesquisa 
configurado de modo a abarcar as especificidades das instituições de microfinanças, principalmente no que tange aos objetivos sociais. Considera que o objetivo principal da instituição é dilatar a abrangência dos seus serviços, o que a faz primar pelo atendimento a um amplo número de clientes, especialmente aqueles considerados menos favorecidos.

Sobre a orientação dos modelos, utlizou-se, em ambos os casos, a DEA com orientação a outputs. Acredita-se que essa especificação apresenta-se apropriada para o caso específico deste trabalho, uma vez que as instituições microfinanceiras necessitam maximizar os seus resultados com o nível de recursos que possuem. lsso porque a maioria das instituições já possui estrutura de recursos limitada (às vezes, restrita por recursos subsidiados) e precisam, com a estrutura existente, maximizar a oferta de serviços, o número de clientes atendidos e os resultados financeiros decorrentes.

No que tange aos retornos de escala, foi empregado o modelo VRS (Retornos Variáveis de Escala), por se considerar que, diante da heterogeneidade de portes e estruturas institucionais no setor microfinanceiro nacional, a hipótese de proporcionalidade entre inputs e outputs subjacente aos modelos CRS (Retornos Constantes de Escala) se mostra muito forte e pouco realística. Além disso, como será detalhado adiante, esse trabalho utiliza uma variável em formato de índice (razão), o que impõe limitações quando do emprego dos modelos CRS, devido à já referida hipótese de proporcionalidade (Hollingsworth \& Smith, 2003; Gomes et al., 2009).

Para seleção das variáveis de inputs e outputs, inicialmente, foi realizada uma lista de variáveis mais importantes empregadas em artigos da literatura específica de $D E A$ aplicada a instituições financeiras e microfinanceiras. Em um segundo momento, foi aplicada a técnica análise fatorial, cujos resultados são explanados na Tabela 2, e então foram escolhidas, dentre as candidatas, duas variáveis de inputs e outputs que apresentaram maiores cargas fatoriais em cada subgrupo.

Assim, despesas operacionais e número de funcionários compuseram, na condição de inputs, ambos os modelos aqui construídos. Em se tratando dos outputs, foram escolhidas as três candidatas de maiores cargas fatoriais, sendo que duas delas (receitas financeiras e carteira ativa) compuseram o modelo de eficiência financeira e a última (número de clientes ativos) foi escolhida para o modelo de eficiência social.

A variável número de clientes foi inserida no modelo de eficiência social para representar a amplitude (breadth) do alcance social da instituição. A profundidade (depth) do alcance (perfil socioeconômico/nível de pobreza da clientela atendida) constitui outro aspecto de suma importância para análise dos resultados sociais das instituições de microfinanças. No entanto, diante de inexistência de dados primários sobre essa variável, recorre-se a

Tabela 2. Análise fatorial das candidatas a variáveis.

\begin{tabular}{|c|c|c|c|c|c|}
\hline \multirow{2}{*}{ Variáveis } & \multicolumn{5}{|c|}{ Fatores } \\
\hline & 1 & 2 & 3 & 4 & 5 \\
\hline Ativo Total & 0,801 & & & & \\
\hline Número de Funcionários & 0,987 & & & & \\
\hline Número de Clientes Ativos & 0,962 & & & & \\
\hline Percentual de Clientes Mulheres & & & $-0,705$ & & \\
\hline Carteira Ativa & 0,946 & & & & \\
\hline Relação Dívida/Capital & & & & & $-0,957$ \\
\hline Retorno sobre Ativo Total & & 0,876 & & & \\
\hline Retorno sobre Patrimônio Líquido & & 0,505 & & & 0,760 \\
\hline Autossuficiência operacional & & 0,878 & & & \\
\hline Margem de Lucro & & 0,853 & & & \\
\hline Custo (despesa) por mutuário & & & $-0,465$ & & \\
\hline Número de Clientes Ativos/Agente de Crédito & & & 0,753 & & \\
\hline Carteira em Risco $>30$ dias & & & & 0,957 & \\
\hline Carteira em Risco $>90$ dias & & & & 0,950 & \\
\hline Receitas Financeiras & 0,979 & & & & \\
\hline Despesas Financeiras & 0,955 & & & & \\
\hline Despesas Operacionais & 0,985 & & & & \\
\hline Capital de Terceiros & 0,757 & & & & \\
\hline Número de agentes de crédito & 0,876 & & & & \\
\hline Despesas Financeiras/Receitas & & $-0,651$ & & & \\
\hline
\end{tabular}

Fonte: Dados da Pesquisa. 
uma proxy para inserir essa informação no modelo de eficiência social.

A literatura apresenta a variável saldo médio de empréstimos por cliente como importante proxy da profundidade do alcance social. Via de regra, o saldo médio de empréstimos é comparado a indicadores de renda (Produto Interno Bruto per capita ou Renda Nacional Bruta per capita), de modo a obter uma métrica que seja relativa às condições econômicas específicas do país (Gutiérrez-Nieto et al., 2009).

Assim, este trabalho adota como proxy da profundidade do alcance social o índice resultante da divisão do saldo médio do empréstimo por cliente pelo Produto Interno Bruto - PIB per capita brasileiro, para o respectivo ano em análise. As informações sobre PIB foram obtidas no acervo de dados on line do Instituto de Brasileiro de Geografia e Estatística - IBGE. Já as informações sobre o valor médio de empréstimos foram coletadas no site THE MIX.

0 trabalho de Gutiérrez-Nieto et al. (2009) foi adotado como referência principal para o cálculo da proxy da profundidade do alcance social. Inicialmente, foram calculados os índices $K_{i}$ a partir da fórmula abaixo:

$$
\mathrm{K}_{\mathrm{i}}=\frac{\text { saldo médio por cliente }}{\text { PIB per capita }}
$$

Na sequência, os valores $K_{i}$ obtidos para cada instituição foram padronizados, resultando em $\mathrm{A}_{i}$.

$$
\mathrm{A}_{\mathrm{i}}=1-\frac{\mathrm{K}_{\mathrm{i}}-\operatorname{Min}(\mathrm{K})}{\text { Amplitude }(\mathrm{K})}
$$

Os valores $A_{i}$ estão, agora, padronizados para ficarem compreendidos entre 0 e 1 , de modo que quanto mais próximo de 1 , maior é o nível de alcance social da instituição.

Os modelos finais de eficiência financeira e social ficaram assim delineados (Tabela 3).

\section{Resultados}

\subsection{Resultados da análise de eficiência}

A Tabela 4 elucida os escores de eficiência alcançados pelas instituições, sob cada um dos modelos, nos três anos de análise (2008, 2009 e 2010), bem como a eficiência média geral para cada especificação em cada um dos anos. Adotar-se-á, doravante, as abreviaturas EF e ES para se referir, respectivamente, a eficiência financeira e eficiência social. As células marcadas com um traço constituem espaços em branco, indicando que, para o ano em questão, não foram encontradas informações referentes à instituição e a mesma não compôs a amostra.
A análise da Tabela 4 evidencia que os escores referentes ao modelo de eficiência financeira apresentam médias muito inferiores àqueles de eficiência social em todos os anos que compuseram o período de análise. No entanto, essa diferença não pode ser comprovada estatisticamente, o que se percebe na Tabela 5, que traz os resultados do Teste Estatístico de Wilcoxon, realizado para testar a igualdade de médias entre eficiência financeira e social. A hipótese nula não pôde ser rejeitada em todos os anos, ao nível de significância de 5\%, sugerindo que as médias de eficiência financeira e social não podem ser consideradas estatisticamente diferentes.

Embora estatisticamente não significativa, a maior eficiência média social indica que existe maior homogeneidade das instituições de microfinanças brasileiras analisadas no que se refere a esse modelo do que quando se trata da eficiência financeira. Em termos de gestão, acredita-se esse resultado sugere certo grau de uniformidade, por parte das instituições pesquisadas, na adoção de estratégias e práticas relativas aos seus resultados sociais. Tal fato, possivelmente, constitui reflexo de um movimento de transparência que ocorre em toda a indústria microfinanceira internacional que enfatiza a importância da adoção de medidas no sentido de gerenciar, avaliar e evidenciar os resultados efetivamente materializados pelas instituições de microcrédito no âmbito social, como elucidam Robinson (2001) e Gutiérrez-Nieto et al. (2009).

Diante do exposto, pode-se dizer que os achados desse trabalho não apontam no sentido de corroborar a tese da corrente welfarista que afirma uma mudança de paradigma no contexto microfinanceiro (Robinson, 2001) que faz com que as instituições se preocupem, com resultados financeiros mais contundentes em detrimento dos resultados sociais. Pelo contrário, o que se pode inferir, a partir dos resultados ora encontrados, é que as instituições de microcrédito brasileiras estão sim focadas na adoção de estratégias e na concretização de resultados ligados à sua missão social.

0 gráfico plotado na Figura 1 evidencia o que aqui se denomina de eficiência dupla das instituições. Cada eixo (horizontal e vertical) representa uma das abordagens construídas neste trabalho (eficiência financeira ou social); os pontos assinalados correspondem ao posicionamento de cada instituição em relação aos níveis de eficiência alcançados considerando os dois modelos, simultaneamente; e as linhas vermelhas traçadas perpendicularmente a cada eixo representam os valores médios de eficiência alcançados pelas instituições na dimensão específica, levando em conta todo o período temporal com dados disponíveis para a unidade em questão. 
Tabela 3. Modelos de eficiência financeira e eficiência social.

\begin{tabular}{|c|c|c|}
\hline Modelo & Variáveis de Inputs & Variáveis de Outputs \\
\hline \multirow{2}{*}{1 - Eficiencia financeira } & Despesas operacionais & Carteira ativa \\
\hline & Número de funcionários & Receitas financeiras \\
\hline \multirow{2}{*}{2 - Eficiencia Social } & Despesas operacionais & Número de clientes ativos \\
\hline & Número de funcionários & Índice de alcance social (proxy) \\
\hline
\end{tabular}

Fonte: Dados da Pesquisa.

Tabela 4. Escores (\%) de eficiência das instituições.

\begin{tabular}{|c|c|c|c|c|c|c|}
\hline & \multicolumn{3}{|c|}{ Eficiência Financeira } & \multicolumn{3}{|c|}{ Eficiência Social } \\
\hline & 2008 & 2009 & 2010 & 2008 & 2009 & 2010 \\
\hline ANDE & 43,7 & 41,6 & & 100,0 & 100,0 & \\
\hline Acredite & 70,4 & 81,6 & & 51,2 & 62,9 & \\
\hline Agência de Crédito & 33,8 & & & 96,2 & & \\
\hline BANCRI & 58,5 & 43,4 & 100,0 & 53,9 & 54,4 & 100,0 \\
\hline Banco da Família & 83,0 & 58,7 & 74,1 & 86,8 & 88,5 & 91,9 \\
\hline Banco do Empreendedor & 87,5 & & & 57,9 & & \\
\hline Banco do Vale & 50,7 & 58,9 & 69,9 & 73,5 & 77,3 & 86,3 \\
\hline CEADe & 47,4 & 37,0 & 51,3 & 54,5 & 84,0 & 92,8 \\
\hline CEAPE BA & 53,4 & 71,1 & 75,8 & 98,4 & 96,6 & 95,0 \\
\hline CEAPE MA & 66,7 & 75,0 & 83,9 & 87,1 & 89,2 & 93,5 \\
\hline CEAPE PE & 55,6 & 40,7 & 52,6 & 86,3 & 82,1 & 91,3 \\
\hline CEAPE Pl & 44,8 & 40,8 & 70,8 & 99,7 & 97,9 & 97,4 \\
\hline CREDIOESTE & 80,8 & 72,3 & 100,0 & 71,0 & 77,0 & 100,0 \\
\hline Casa do Microcrédito & 99,8 & & & 60,9 & & \\
\hline Central Cresol Baser & 100,0 & 100,0 & 100,0 & 32,2 & 28,4 & 23,1 \\
\hline CrediAmigo & 100,0 & 100,0 & 100,0 & 100,0 & 100,0 & 100,0 \\
\hline Credisol & 99,4 & 100,0 & 100,0 & 61,4 & 47,4 & 49,2 \\
\hline Credito Solidário & 30,1 & 25,7 & 51,6 & 96,6 & 96,9 & 99,9 \\
\hline Cresol Central & 100,0 & 100,0 & 100,0 & 100,0 & 78,8 & 72,8 \\
\hline Fácil SCM & 93,3 & 100,0 & & 27,3 & 40,4 & \\
\hline 1CC BluSol & 81,5 & 76,2 & 85,8 & 65,1 & 73,7 & 89,2 \\
\hline ICC Conquista Solidária & 64,3 & & & 100,0 & & \\
\hline 1CC MAUCE & 100,0 & 100,0 & & 100,0 & 100,0 & \\
\hline Instituto Estrela & 38,0 & 21,9 & 100,0 & 100,0 & 100,0 & 100,0 \\
\hline Real Microcrédito & 74,7 & 64,2 & 71,8 & 100,0 & 100,0 & 100,0 \\
\hline Rede Novo Sol & 100,0 & & & 100,0 & & \\
\hline SOCIALCRED & 100,0 & 84,9 & & 44,1 & 11,3 & \\
\hline SOCRED & 100,0 & & & 100,0 & & \\
\hline São Paulo Confia & 54,2 & 41,3 & 55,5 & 95,4 & 95,2 & 95,9 \\
\hline Média & 72,8 & 64,8 & 78,4 & 79,3 & 77,5 & 87,7 \\
\hline
\end{tabular}

Fonte: Dados da Pesquisa.

Tabela 5. Resultados teste Wilcoxon EF e ES.

\begin{tabular}{cccc}
\hline & Ano & Valor Z & Significância \\
\hline EF - ES & 2008 & $-0,914$ & 0,360 \\
EF - ES & 2009 & $-1,216$ & 0,223 \\
EF - ES & 2010 & $-1,224$ & 0,220 \\
\hline
\end{tabular}

Fonte: Dados da Pesquisa.

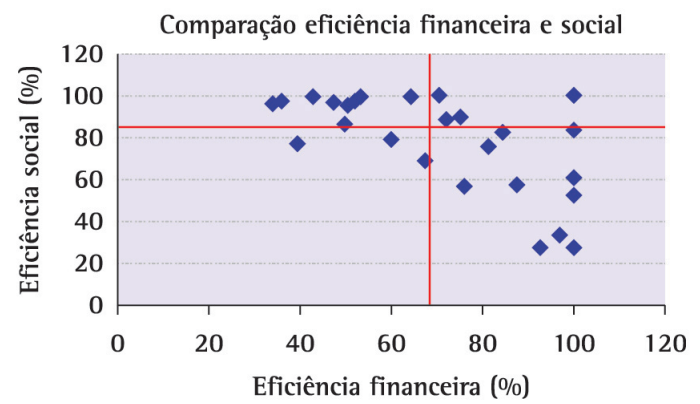

Figura 1. Comparação eficiência financeira e eficiência social. Fonte: Dados da Pesquisa.
Nota-se, inicialmente, que os escores de eficiência possuem valores médios relativamente altos $(72,0$ e 81,5 , respectivamente para eficiência financeira e social, para o período de três anos), bem como consideravelmente alta dispersão dos valores da série, o que se pode notar pelos coeficientes de variação de $31,48 \%$ e $28,46 \%$, para eficiência financeira e social, respectivamente.

A localização das instituições suscita algumas considerações sobre a performance das mesmas. No quadrante superior direito estão situadas as instituições que obtiveram níveis de eficiência relativa superiores à média em ambos os quesitos, ou seja, financeiro e social, podendo ser consideradas as "unidades destaque" em se tratando das dimensões de eficiência avaliadas. Exemplos de instituições nessa condição são Crediamigo, Cresol Central, ICC MAUCE e Socred. Algumas dessas instituições atuam em grandes escalas em termos de carteira ativa e receitas financeiras 
(outputs considerados). Outras, embora possuam carteiras ativas menores, também alcançaram a fronteira de eficiência financeira devido ao pequeno número de funcionários e nível reduzido de despesas operacionais (inputs) com que conseguem operar.

No que ser refere à eficiência social, no intuito de alcançar a fronteira, as instituições de maior porte como Crediamigo e Cresol Central atribuíram pesos mais significativos ao output número de clientes ativos e ao input despesas operacionais. Já nas instituições de menor porte, a exemplo, Instituto Estrela e ICC-MAUCE, os altos níveis de eficiência foram obtidos com a maior influência das variáveis alcance social como output e número de funcionários como input, variáveis essas que contribuíram de forma mais incisiva para a obtenção dos níveis de eficiência apresentados na Tabela 4.

Especialmente, acredita-se que o foco estratégico em economias de escala e racionamento de gastos operacionais pode constituir diferencial importante dos programas de microcrédito exitosos nesses dois quesitos, e que podem inspirar práticas de outras instituições, estabelecendo importantes benchmarks que podem aprimorar as estratégias de gestão da indústria microfinanceira como um todo.

0 quadrante superior esquerdo agrega as instituições com bons níveis de desempenho social e menos eficientes no que se refere à esfera financeira. As unidades situadas nesse quadrante, possivelmente, precisam implementar ações no sentido de aumentar suas receitas financeiras e, principalmente, suas carteiras ativas, de modo a avançarem para maiores níveis de eficiência financeira.

Por seu turno, as instituições situadas no quadrante inferior direito apresentam eminente êxito financeiro, mas, por outro lado, desempenho limitado na esfera social. Uma análise desses resultados paralelamente aos pesos atribuídos a inputs/ outputs fornece indícios de que a trajetória dessas instituições para uma maior eficiência social passa pela ampliação do número de clientes ativos e, principalmente, pelo aprofundamento do alcance social.

Finalmente, as instituições situadas no quadrante inferior esquerdo possuem as piores performances dentre as instituições avaliadas, conjugando baixos níveis de desempenho financeiro e social. Essas instituições necessitam imprimir melhorias tanto nas variáveis relacionadas a resultado financeiro (carteira ativa e receitas financeiras) quanto naquelas que delimitam o modelo social (número de clientes ativos e alcance social). Ressalte-se que é um pequeno número de instituições que se encontra nessa situação e, além disso, mesmo situando no quadrante de pior resultado, a análise gráfica evidencia que o desempenho desse grupo de instituições não se desvia muito na média, embora seja inferior a ela em todos os casos, justificando a sua plotagem nesse quadrante.

\subsection{Indice Malmquist de produtividade}

No contexto de aplicação da $D E A$, quando se trabalha com dados referentes a mais de um período temporal, é freqüente o emprego do Índice Malmquist para medir a mudança de eficiência e produtividade ao longo do tempo.

Segundo Ferreira \& Gomes (2009), o Índice de Malmquist foi inicialmente proposto por Malmquist (1953) para analisar questões relacionadas ao consumo. Caves et al. (1982) adaptaram-no para o contexto de análise de produção. No entanto, é atribuído a Färe et al. (1994) o reconhecimento de que a função distância implícita no Índice de Malmquist era recíproca da medida de eficiência técnica proposta por Farrel (1957). Assim, esses autores utilizaram a programação linear que embasa a $D E A$ para o cálculo do índice de variações de produtividade.

Segundo Cook \& Zhu (2008), o Índice de Malmquist tem se mostrado um instrumento adequado para aferir mudanças de eficiência/produtividade de $D M U$ s analisadas através da $D E A$. Ainda segundo os autores, uma das grandes vantagens do índice reside em sua característica fundamental de ser decomposto em dois componentes, um que fornece a medida das mudanças da fronteira técnica/tecnológica e outro que mensura a mudança na eficiência técnica propriamente dita.

De acordo com Cook \& Zhu (2008) e Ferreira \& Gomes (2009), o índice de produtividade total de Malmquist $\mathrm{M}_{0}$ orientado a outputs, usado para analisar a variação produtiva entre os períodos $\mathrm{t} e$ $\mathrm{t}+1$, é definido da seguinte forma:

$$
M_{o}=\frac{\theta_{o}^{t+1}\left(\mathrm{x}_{\mathrm{o}}^{\mathrm{t}+1}, y_{o}^{t+1}\right)}{\theta_{o}^{t}\left(\mathrm{x}_{\mathrm{o}}^{\mathrm{t}}, y_{o}^{t}\right)} \times\left[\frac{\theta_{o}^{t}\left(\mathrm{x}_{\mathrm{o}}^{\mathrm{t}+1}, y_{o}^{t+1}\right)}{\theta_{o}^{t+1}\left(\mathrm{x}_{\mathrm{o}}^{\mathrm{t}+1}, y_{o}^{t+1}\right)} \frac{\theta_{o}^{t}\left(\mathrm{x}_{\mathrm{o}}^{\mathrm{t}}, y_{o}^{t}\right)}{\theta_{o}^{t+1}\left(\mathrm{x}_{\mathrm{o}}^{\mathrm{t}}, y_{o}^{t}\right)}\right]^{1 / 2}
$$

onde:

$\mathrm{M}_{\mathrm{o}}$ é o Índice de Malmquist a ser calculado; $\mathrm{x}_{\mathrm{io}}$ e $\mathrm{y}_{\mathrm{jo}}$ são os inputs $i$ e outputs $j$ da DMU em análise; e $\theta_{0}^{t}\left(x_{0}^{t}, y_{0}^{t}\right) \theta_{0}^{t+1}\left(x_{0}^{t+1}, y_{0}^{t+1}\right)$ representam a eficiência técnica da referida $D M U$ nos tempos t e $t+1$, respectivamente.

A expressão de cálculo do Índice de Malmquist acima exposta encontra-se em sua forma decomposta, ou seja, isolando a variação de eficiência técnica do deslocamento da fronteira tecnológica. Nesse sentido, o termo à esquerda, externo ao colchete, evidencia a comparação da eficiência técnica entre dois períodos de tempo, denominada emparelhamento. E o termo interno ao colchete refere-se ao deslocamento da 
fronteira tecnológica. 0 índice de Malmquist é, na verdade, composto pelo produto desses dois termos.

A respeito desses termos componentes do Índice de Malmquist, Ferreira \& Gomes (2009) elucidam que a variação de eficiência técnica ou emparelhamento é determinada pelos escores de eficiência técnica que, por sua vez, são medidos pelas distâncias das $D M U s$ às suas respectivas fronteiras eficientes. No entanto, os avanços de produtividade de uma $D M U$ podem resultar também de inovações ou progresso tecnológico, podendo ocorrer um deslocamento da fronteira, em razão da introdução de tecnologias mais avançadas. Disso decorre a inserção de ambos os itens no cálculo da variação da produtividade/ eficiência ao longo do tempo.

$\mathrm{Na}$ orientação a outputs (adotada neste trabalho), prevalecem as seguintes condições para o Índice de Malmquist, emparelhamento e fronteira tecnológica (Ferreira \& Gomes, 2009):

- Índice de Malmquist $\left(\mathrm{M}_{\mathrm{o}}\right)$ maior que 1, igual a 1, menor que 1: significa, respectivamente, aumento de produtividade, produtividade constante e declínio de produtividade entre os períodos $t$ e $t+1$;

- Índice de Emparelhamento maior que 1, igual a 1, menor que 1: significa, respectivamente, melhoria de eficiência técnica, eficiência técnica constante e piora de eficiência técnica entre os períodos t e $t+1$;

- Índice de Variação Tecnológica maior que 1 , igual a 1, menor que 1: significa, respectivamente, progresso tecnológico, constância das condições tecnológicas, e regressão tecnológica entre os períodos t e $t+1$.

A partir de uma base de dados conjunta, construída de modo a abarcar os três anos sob análise, calculou-se o Índice de Malmquist e os indicadores de variação de eficiência técnica e tecnológica que o compõem, utilizando, para tal, o software Frontier Analyst ${ }^{\circledR}$. A Tabela 6 exibe os valores médios obtidos para cada um desses índices calculados.

$\mathrm{Na}$ abordagem financeira, verifica-se uma tendência de aumento de produtividade ao longo dos três anos analisados. 0 índice de Malmquist evidencia ligeiro aumento de produtividade no período de 2008 para 2009, tendência essa que se torna mais vigorosa para o período 2009/2010. No entanto, esse aumento de produtividade não constitui em sua totalidade, reflexo da melhoria no nível de eficiência técnica das instituições ao longo do tempo. Na verdade, a eficiência técnica teve peso significativo no aumento da produtividade apenas no período 2009/2010. Para o período 2008/2009, o acréscimo do Índice de Malmquist não pode ser atribuído à eficiência técnica, que sofreu redução nesse período, mas sim a um deslocamento da fronteira tecnológica, denotando prováveis mudanças tecnológicas positivas que afetam o setor microfinanceiro como um todo.

Em se tratando da abordagem social, os valores do Índice de Malmquist também evidenciam um aumento de produtividade ao longo de todo período. De 2008 para 2009, essa melhoria de produtividade pode ser atribuída, principalmente, à eficiência técnica, que sofreu considerável aumento nesse período, movimentando-se em sentido oposto à fronteira tecnológica, que se deslocou negativamente, ou seja, regressão tecnológica.

Já no período 2009/2010 a variação positiva da eficiência técnica é reforçada pelo progresso (deslocamento positivo) da fronteira tecnológica, e esses dois movimentos confluem para uma variação positiva no Índice de Malmquist, evidenciando, assim, aumento de produtividade na abordagem social também nesse período.

Assim, a análise do índice de Malmquist revela, em ambas as abordagens, uma tendência de variação temporal que pode ser qualificada como ascendente. Ou seja, existe tendência de variação positiva da produtividade ao longo do período analisado tanto sob a ótica financeira quanto social. No entanto, enquanto na abordagem social essa variação positiva constitui, quase que exclusivamente, reflexo de acréscimos na eficiência técnica, na abordagem financeira nota-se também uma grande sensibilidade à variação da fronteira tecnológica, que impacta significativamente sobre o Índice de Malmquist.

Acredita-se que essas variações da fronteira tecnológica podem estar associadas a mudanças estruturais que se fazem notar no setor de microcrédito brasileiro, principalmente nos últimos anos, em que essa modalidade de crédito tem sido foco de atenção do governo, organismos multilaterais e instituições financeiras como um todo. Algumas estratégias recentes emanantes desses e outros stakeholders acabam por demandar mudanças, imputando novas técnicas e tecnologias de gestão em todo o setor.

Tabela 6. Índice de Malmquist.

\begin{tabular}{lcccc}
\hline & \multicolumn{2}{c}{ Abordagem Financeira } & \multicolumn{2}{c}{ Abordagem Social } \\
\cline { 2 - 4 } & $2008 / 2009$ & $2009 / 2010$ & $2008 / 2009$ & $2009 / 2010$ \\
\hline İndice de Malmquist & 1,0405 & 1,0641 & 1,0333 & 1,0436 \\
Variação Eficiência Técnica & 0,8332 & 1,4683 & 1,0613 & 1,0294 \\
Variação Fronteira Tecnológica & 1,2610 & 0,8229 & 0,9871 & 1,0205 \\
\hline
\end{tabular}

Fonte: Dados da Pesquisa. 
Os resultados encontrados permitem inferir que tais estratégias produzem efeitos positivos e negativos em ambas as abordagens, que apresentam, durante o período analisado, tanto regressão (deslocamento negativo) quanto progresso (deslocamento positivo) da fronteira tecnológica. Os deslocamentos da fronteira sob a perspectiva financeira são mais acentuados, enquanto que sob o enfoque social ocorrem apenas variações pouco significativas.

Em suma, a análise de eficiência/produtividade ao longo do tempo corrobora as inferências realizadas anteriormente a partir dos escores médios de eficiência e indica que há uma maior homogeneidade das instituições analisadas no que se refere aos resultados de eficiência social comparativamente à financeira. As variações de produtividade sob o enfoque financeiro parecem refletir mudanças tecnológicas no setor, enquanto que, no âmbito social, nota-se uma maior constância de evolução da eficiência técnica social e menor influência das variações na fronteira tecnológica.

\section{Considerações finais}

De modo geral, os resultados encontrados no artigo evidenciam, na análise de cada ano individualmente, maiores escores de eficiência social comparativamente à eficiência financeira. $\mathrm{Na}$ análise realizada ao longo de todo o período temporal considerado, através do Índice de Malmquist, percebeu-se uma evolução da produtividade em ambas as abordagens. No entanto, na abordagem social, a evolução de produtividade, reflete, em maior grau, variações positivas efetivas da eficiência técnica, enquanto que na abordagem financeira foi notório o impacto dos deslocamentos na fronteira tecnológica.

Esses achados sugerem que, de modo semelhante ao que ocorre no setor microfinanceiro internacional, também as instituições brasileiras adotam posturas mais coesas na adoção de práticas de gestão relacionadas à concretização e evidenciação de seus resultados sociais quando cotejados com aqueles financeiros. Tal fato reflete, acredita-se, um isomorfismo institucional que ocorre, em grande parte, devido à pressão de stakeholders diversos (doadores, organismos multilaterais, investidores sociais, organismos reguladores etc.) que passam a cobrar, cada vez mais, que as instituições adotem uma postura estratégica de gerenciamento do desempenho social, tal como já fazem com o financeiro, bem como evidenciem os resultados efetivamente concretizados nesse campo (Robinson, 2001).

Sobre esses resultados supramencionados, a grande questão que se coloca é em que medida o foco em estratégias e resultados sociais pode ser conciliado à autonomia e sustentabilidade financeira da instituição. Em outras palavras, é possível concretizar a missão social de contribuir para o combate à pobreza e ainda lograr resultados financeiros positivos, que propiciem a sustentabilidade da instituição na esfera financeira, sem depender de recursos financeiros subsidiados? 0 desempenho social, em si, é um tanto positivo, e a sua busca constitui estratégia necessária, uma vez que se vincula às origens do microcrédito. No entanto, muitas vezes, a materialização da missão social tem como preço a perda da autonomia financeira. Para aprofundar seu alcance social através da atuação com público-alvo socioeconomicamente menos favorecido, muitas vezes, as instituições de microcrédito deixam de adotar medidas necessárias à consolidação da autonomia/sustentabilidade financeira (por exemplo, ampliação de carteiras ativas, maximização de receitas, aumentos de taxas de juros, aumento de valores de empréstimos etc.).

A análise dupla de eficiência aponta que existem, no contexto brasileiro estudado, instituições que conseguem conciliar a eficiência financeira com a social, apresentando-se como possiveis benchmarks para a indústria microfinanceira nacional. Em algumas dessas instituições, a atuação com carteiras ativas não necessariamente exclui a orientação institucional a clientes socioeconomicamente mais vulneráveis. Essa questão suscita que as estratégias implementadas pelas instituições que conseguem o feito de conciliar amplitude da carteira e profundidade do alcance social possam ser utilizadas como referência para as outras instituições do setor, delineando possíveis benchmarks para o setor microfinanceiro brasileiro.

\section{Referências}

Alves, S., \& Soares, M. (2004). Democratização do crédito no Brasil: atuação do Banco Central. Brasília: Banco Central do Brasil.

Annim, S. K. (2010). Microfinance efficiency trade-offs and complementarities (Working Paper 127). Manchester: World Poverty Institute.

Banker, R., Charnes, A., \& Cooper, W. W. (1984). Some models for estimating technical and scale inefficiencies in data envelopment analysis. Management Science, 30(9), 1078-1092. http://dx.doi.org/10.1287/mnsc.30.9.1078

Barone, F., Lima, P., Dantas, V., \& Rezende, V. (2002). Introdução ao microcrédito. Brasília: Conselho da Comunidade Solidária.

Berger, A. N., \& Humphrey, D. B. (1997). Efficiency of financial institutions: international survey and directions for future research. Europeran Journal of Operational Research, 98(2), 175-212. http://dx.doi.org/10.1016/ S0377-2217(96)00342-6

Braga, M. B., \& Toneto Junior, R. (2000). Microcrédito: aspectos teóricos e experiências. Análise Econômica, 18(33), 69-86.

Carneiro, J. M. T., Silva, J. F., Rocha, A., \& Hemais, C. (2005, Junho). Mensuração do desempenho organizacional: questões conceituais e metodológicas. In Anais do Encontro de Estudos em Estratégia, Rio de Janeiro, Brasil. 
Caves, D. W., Christensen, L. R., \& Diewert, W. E. (1982). The economic theory of index numbers and the measurement of input, output and productivity. Econometrica, 50(6), 1393-1414. http://dx.doi. org $/ 10.2307 / 1913388$

Charnes, A., Cooper, W. W., \& Rhodes, E. (1978). Measuring the efficiency of decision making units. European Journal of Operational Research, 2(6), 429-444. http:// dx.doi.org/10.1016/0377-2217(78)90138-8

Cook, W. D., \& Zhu, J. (2008). Data envelopment analysis: modeling operational processes and measuring productivity. Dordrecth: Kluwer Academic Publishers.

Ejigu, L. (2009). Performance analysis of a sample of microfinance institutions of Ethiopia. International NGO Journal, 4(5), 287-298.

Färe, R., Grosskopf, S., Norris, M., \& Zhang, Z. (1994). Productivity growth, technical progress, and efficiency change in industrialized countries. American Economic Review, 84(1), 66-83.

Farrel, M. J. (1957). The measurement of productive efficiency. Journal of the Royal Statistical Society, 120(3), 253-290. http://dx.doi.org/10.2307/2343100

Ferreira, C. M., \& Gomes, A. P. (2009). Introdução à análise envoltória de dados: teoria, modelos e aplicações. Viçosa: UFV.

Gomes, E. G., Grego, C. R., Mello, J. C. C. B. S., Valladares, G. S., Mangabeira, J. A. C., \& Miranda, E. E. (2009). Dependência espacial da eficiência do uso da terra em assentamento rural na Amazônia. Produção, 19(2), 417-432. http:// dx.doi.org/10.1590/S0103-65132009000200015

Gonzalez-Vega, C. (1986). Mercados financieros y desarrollo. Santo Domingo: Centro de Estudios Monetarios y Bancários.

Gutiérrez-Nieto, B., Serrano-Cinca, C., \& Molinero, C. M. (2007) Microfinance institutions and efficiency. The International Journal of Management Science, 35(2), 131-142.

Gutiérrez-Nieto, B., Serrano-Cinca, C., \& Molinero, C. M. (2009). Social efficiency in microfinance institutions. Journal of Operational Research Society, 60(1), 104-119. http://dx.doi.org/10.1057/palgrave.jors.2602527

Hollingsworth, G., \& Smith, P. (2003). Use of ratios in data envelopment analysis. Applied
Economics Letters, 10(11), 733-735. http://dx.doi. org/10.1080/1350485032000133381

Kaplan, R. S., \& Norton, D. (1997). A estratégia em ação: balanced scorecard (6th ed.). Rio de Janeiro: Campus

Lins, M. P. E., \& Meza, L. A. (2000). Análise envoltória de dados e perspectivas de integração no ambiente do apoio à decisão. Rio de Janeiro: UFRJ.

Malmquist, S. (1953). Index numbers and indifference curves. Trabajos de Estatistica, 4(1), 209-242. http://dx.doi. org/10.1007/BF03006863

Mello, J. C. C. B. S., Meza, L. A., Gomes, E. G., \& Biondi Neto, L. (2005, Setembro). Curso de análise envoltória de dados. In Anais do Simpósio Brasileiro de Pesquisa Operacional, Gramado, Brasil.

Microfinance Information Exchange. (2010). Brasil 2009 Informativo e análise das microfinanças. Rio de Janeiro: TheMix/FGV Small Business.

Naqvi, F. B., \& Guzmán, G. F. (2003/2004) Microfinanças em foco. Revista de Administração de Empresas, 2(4), 25-29.

Nichter, S., Goldmark, L., \& Fiori, A. (2002). Entendendo as microfinanças no contexto brasileiro. Rio de Janeiro: BNDES.

Niederauer, C. A. P. (1988). Avaliação dos bolsistas em produtividade da engenharia de produção usando Análise Envoltória de Dados (Dissertação de mestrado). Universidade Federal de Santa Catarina, Florianópolis. PMid:3164671.

Parente, S. (2002). Microfinanças: saiba o que é um banco do povo. Brasília: Agência de Educação para o Desenvolvimento.

Ribeiro, C. T., \& Carvalho, C. E. (2006). Do microcrédito às microfinanças: desempenho financeiro, dependência de subsídios e fontes de financiamento: uma contribuição à análise. São Paulo: EDUC.

Robinson, M. (2001). The microfinance revolution: sustainable finance for the poor. Washington: The World Bank. http://dx.doi.org/10.1596/0-8213-4524-9

Soares, M., \& Melo Sobrinho, A. (2008). Microfinanças: o papel do Banco Central do Brasil e a importância do cooperativismo de crédito (2. ed). Brasília: Banco Central do Brasil.

Yunus, M. (2002). O banqueiro dos pobres. São Paulo: Ática.

\title{
Efficiency of Microcredit Institutions: an application of DEA/VRS to the Brazilian case
}

\begin{abstract}
The purpose of this article was to evaluate the efficiency of microfinance institutions in Brazil under two distinct approaches: financial and social. In terms of methodology, we used the technique of linear programming Data Envelopment Analysis - DEA applied to a sample of institutions listed in THE MIX database in the period 2008, 2009 and 2010, and Malmquist Index for analysis of changes in productivity and efficiency of the institutions over time. The results show higher levels of social efficiency compared to the financial for each year individually. The temporal analysis revealed an increase in productivity under both approaches. This increase reflected actual changes in technical efficiency in a greater extent in the social model and shifts of technological frontier in the financial model. Overall, these results indicate a greater homogeneity of the institutions with regard to strategies and practices related to social efficiency when compared with financial.
\end{abstract}

\section{Keywords}

Finance. Microcredit. Economic development. Efficiency. Data Envelopment Analysis. 